\title{
Language development and the ontogeny of the dorsal pathway
}

\author{
Angela D. Friederici * \\ Department of Neuropsychology, Max Planck Institute for Human Cognitive and Brain Sciences, Leipzig, Germany
}

Edited by:

Constance Scharff, Freie Universitaet Berlin, Germany

\section{Reviewed by:}

Steven Chance, Oxford University, UK Paul M. Nealen, Indiana University of Pennsylvania, USA

${ }^{*}$ Correspondence:

Angela D. Friederici, Department of Neuropsychology, Max Planck

Institute for Human Cognitive and

Brain Sciences, Stephanstraße 1A,

04103 Leipzig, Germany.

e-mail: angelafr@cbs.mpg.de
In the absence of clear phylogenetic data on the neurobiological basis of the evolution of language, comparative studies across species and across ontogenetic stages within humans may inform us about the possible neural prerequisites of language. In the adult human brain, language-relevant regions located in the frontal and temporal cortex are connected via different fiber tracts: ventral and dorsal pathways. Ontogenetically, it has been shown that newborns display an adult-like ventral pathway at birth. The dorsal pathway, however, seems to display two subparts which mature at different rates: one part, connecting the temporal cortex to the premotor cortex, is present at birth, whereas the other part, connecting the temporal cortex to Broca's area, develops much later and is still not fully matured at the age of seven. At this age, typically developing children still have problems in processing syntactically complex sentences. We therefore suggest that the mastery of complex syntax, which is at the core of human language, crucially depends on the full maturation of the fiber connection between the temporal cortex and Broca's area.

Keywords: grammar, development, fiber tract, arcuate fasciculus
The neural basis of language evolution must remain speculative, since clear phylogenetic data are unavailable. However, there are two alternative, though more indirect ways, to approach this issue. One approach is to compare different species in their ability to learn language, in particular, syntax or rule-based sequences. A second is to consult ontogenetic data on language development and brain maturation, under the assumption that ontogeny to some extent reflects phylogeny. In this article, data from both approaches, with a strong focus on rule-based and syntactic sequence learning, will be discussed.

Central to the discussion is not only whether such sequences can be learned, but more crucially, what type of syntactic sequence can be learned. A fundamental distinction has been made between two grammar types, namely finite state grammars (FSG) following an $(\mathrm{AB})^{n}$ rule and phrase structure grammar (PSG) following an $\mathrm{A}^{n} \mathrm{~B}^{n}$ rule (Hauser et al., 2002; Fitch and Hauser, 2004; see Figure 1).

There are at least three possible mechanisms through which grammatical sequence learning can take place: (1) adjacent dependencies, as in $(\mathrm{AB})^{n}$ grammars, and also non-adjacent dependencies, which do not involve higher-order hierarchies, could be learned by extracting phonological regularities from the auditory input and memorizing these for further use; (2) adjacent dependencies between $\mathrm{A}$ and $\mathrm{B}$ in $(\mathrm{AB})^{n}$ grammars or between a determiner, e.g., the and a noun, e.g., man in natural grammars could be learned through the same mechanism described in (1), but without the buildup of a minimal hierarchy or (3) through the computation "Merge" that binds two elements into a minimal hierarchical structure (Chomsky, 1995) the basic mechanism to learn a natural grammar with its asymmetric higher-order hierarchical structure. This requires a computational system that goes beyond the mechanisms described in (1) and (2), and requires the computation Merge more than once.

In the following section, we will review the success of grammar learning in different species, discuss the possible underlying processing mechanisms, and debate their neural basis. The data from these studies examining this suggest that the three grammar learning mechanisms described above can be related to three different neural circuits: (1) an input-to-output circuit present in vocal learning animals, (2) a circuit subserving the learning of $(\mathrm{AB})^{n}$ structures, and (3) a circuit involving the learning of $\mathrm{A}^{n} \mathrm{~B}^{n}$ structures.

\section{GRAMMAR LEARNING ACROSS SPECIES}

There are several studies that have taken a species-comparative approach. Some have compared artificial grammar learning between human and non-human primates, or have used similar grammar types to investigate songbirds' ability to learn grammatical sequences. Others have additionally discussed the neural basis of these learning abilities.

Fitch and Hauser (2004) were the first to investigate grammar learning in human and non-human primates using FSG and PSG grammars (Figure 1). Testing cotton-top tamarins and human adults in a behavioral grammar learning study, they found that humans could learn both grammar types easily, whereas the monkeys were only able to learn the FSG. The neural basis for this ability in cotton-top tamarins is unknown, since there are no functional or structural brain studies on this type of monkey. There are, however, a number of structural imaging studies on macaques, chimpanzees, and humans (Catani et al., 2002; Anwander et al., 2007; Rilling et al., 2008; Saur et al., 2008; Makris and Pandya, 2009; Petrides and Pandya, 2009). These studies indicate that the frontal and temporal regions which are known to be involved 


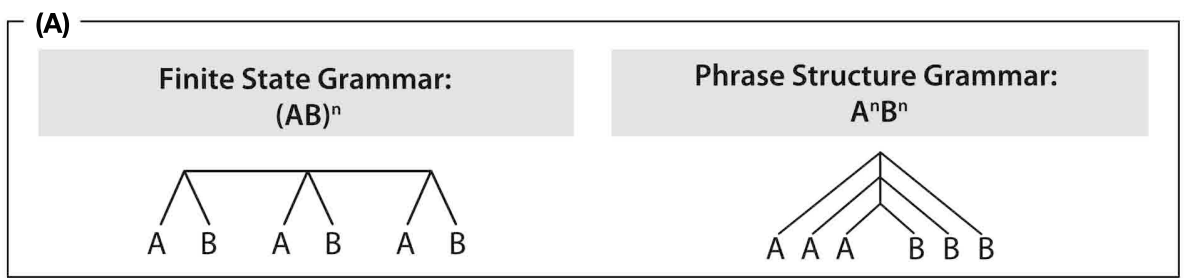

- (B) Examples of stimulus items

Category A: pa, li, mo, nu, ka, bi, do, gu

la pa wu mo no li
Category B: ba, di, yo, tu, la, mi, no, wu ba la tu li pa ka

Adapted from Fitch \& Hauser, Science, 2004

FIGURE 1 | Artificial grammar used in Fitch and Hauser (2004). (A) Structure of sequences. (B) Category A syllables and Category B syllables used in the sequences as well as examples of an $(A B)^{n}$ sequence (left panel) and an $\mathrm{A}^{n} \mathrm{~B}^{n}$ sequence (right panel). Category $\mathrm{A}$ syllables were produced by a female speaker, Category B syllables by a male speaker. Category membership was thus coded by the pitch of voice. in language processing in humans are connected via ventral and dorsal fiber bundles in both humans and non-human primates. A direct comparison, however, revealed differences between humans and non-human primates; macaques and chimpanzees display a strong ventral and a weak dorsal pathway, whereas humans display a strong dorsal pathway and a well-developed ventral pathway. The dorsal pathway was, therefore, discussed as the crucial pathway for the language ability in humans (Rilling et al., 2008).

This difference in the structure of these pathways between humans and non-human primates is of particular interest in the light of a functional and structural imaging study in humans (Friederici et al., 2006), which applied the same artificial grammar types as used in the behavioral study by Fitch and Hauser (2004). In humans, the $(\mathrm{AB})^{n}$ grammar, with its adjacent dependencies, activated the frontal operculum, which is connected via the ventral pathway to the temporal cortex. Interestingly, the $\mathrm{A}^{n} \mathrm{~B}^{n}$ grammar additionally recruited Broca's area, which is connected to the temporal cortex via the dorsal pathway (Friederici et al., 2006). These data were taken to suggest that Broca's area and its dorsal connection to the temporal cortex, in particular, supports the processing of higher-order hierarchically structured sequences relevant to language.

This conclusion, however, was challenged on both theoretical and empirical grounds. It has been argued that the processing of $\mathrm{A}^{n} \mathrm{~B}^{n}$ grammar does not necessarily require the buildup of a hierarchical structure, but could be based on a simpler computation involving a counting mechanism plus some memory abilities (Perruchet and Rey, 2005; de Vries et al., 2008). The empirical challenge comes from studies reporting that songbirds are able to process $\mathrm{A}^{n} \mathrm{~B}^{n}$ grammars (Gentner et al., 2006; Abe and Watanabe, 2011). However, although the grammar used by Abe and Watanabe (2011) can be described as being asymmetric, similar to natural languages, the detection of the incorrect sequences in the experiment could, in principle, be performed based on the following computation: process the incoming sequence of (adjacent) elements and, upon detection of the center element, reverse-and-match the following sequence to the

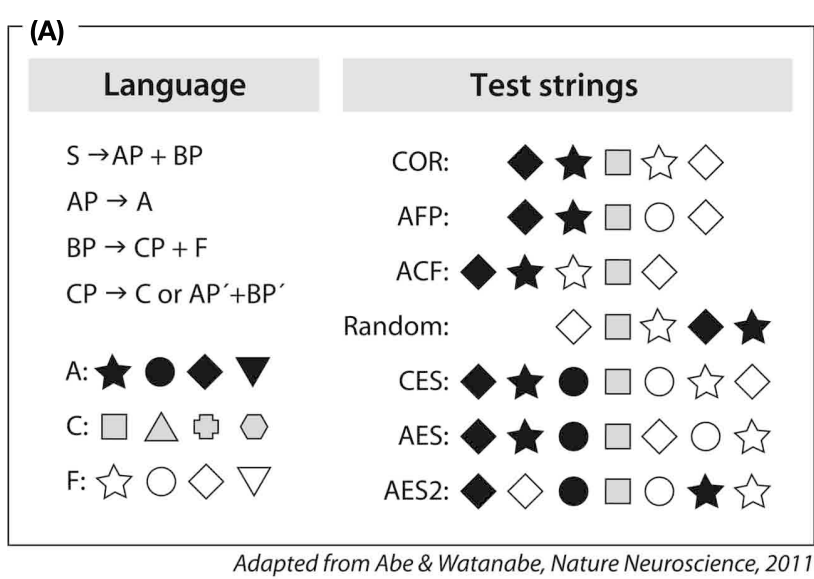

[B) Example structures of test strings

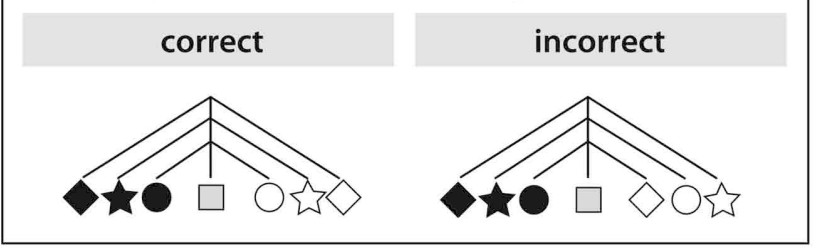

FIGURE 2 | Artificial grammar used in Abe and Watanabe (2011). (A) Left panel: description of the grammar as a center-embedded structure and members of categories A, C, and F; Right panel: examples of test strings. (B) Symmetrical description of center-embedded structure (CES) and violating test string (AES). The center element is represented in gray.

initial sequence (Figure 2). The underlying mechanisms used to process symmetrical $\mathrm{A}^{n} \mathrm{~B}^{n}$ grammars thus remain speculative, both for the songbird studies (Gentner et al., 2006; Abe and Watanabe, 2011) and for the human study (Friederici et al., 2006).

For humans, however, the argument can be made that the computation they apply to process symmetrical $\mathrm{A}^{n} \mathrm{~B}^{n}$ grammars does indeed involve hierarchy building. The argument is 
based on two findings. Humans process symmetrical grammatical structures lacking functional categories (Friederici et al., 2006; Bahlmann et al., 2008) and also asymmetrical natural grammars, which require the buildup of a multi-level hierarchy, using the same brain area; namely Broca's area (Makuuchi et al., 2009; see Figure 3). This brain region is part of the neural network which is dorsally connected to the temporal cortex via the superior longitudinal fascile and the arcuate fascile (Friederici et al., 2006). The finding that humans process symmetrical structures of artificial $\mathrm{A}^{n} \mathrm{~B}^{n}$ grammars within the same brain region used to process hierarchical asymmetrical structures in natural language leads to the conclusion that the underlying mechanism for both is that of building hierarchies.

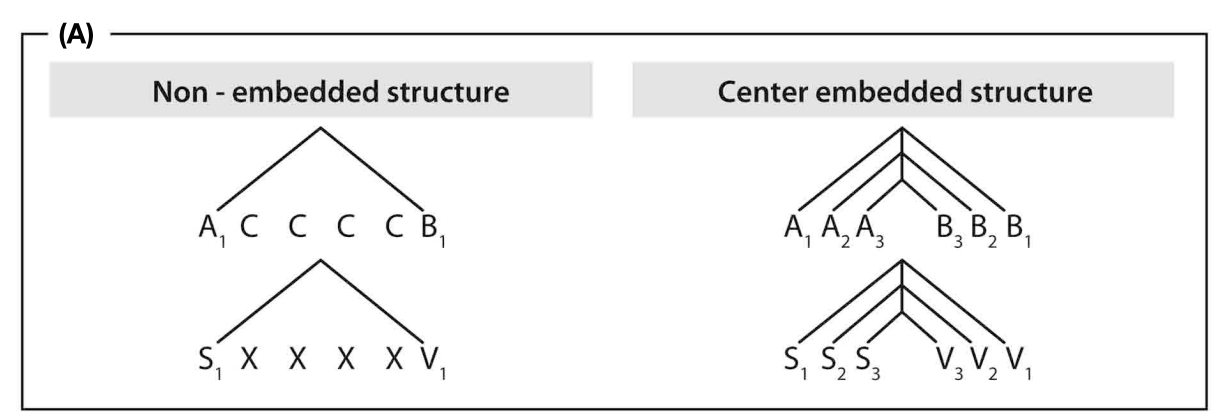

(B) Examples of stimulus items

Peter wusste, dass ...

Peter knew that ...

[Maria, [die Hans, [der gut aussah], liebte], Johann geküsst hatte.]

Maria who loved Hans who was good looking had kissed Johann.

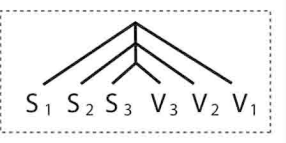

Adapted from Makuuchi et al., PNAS, 2009

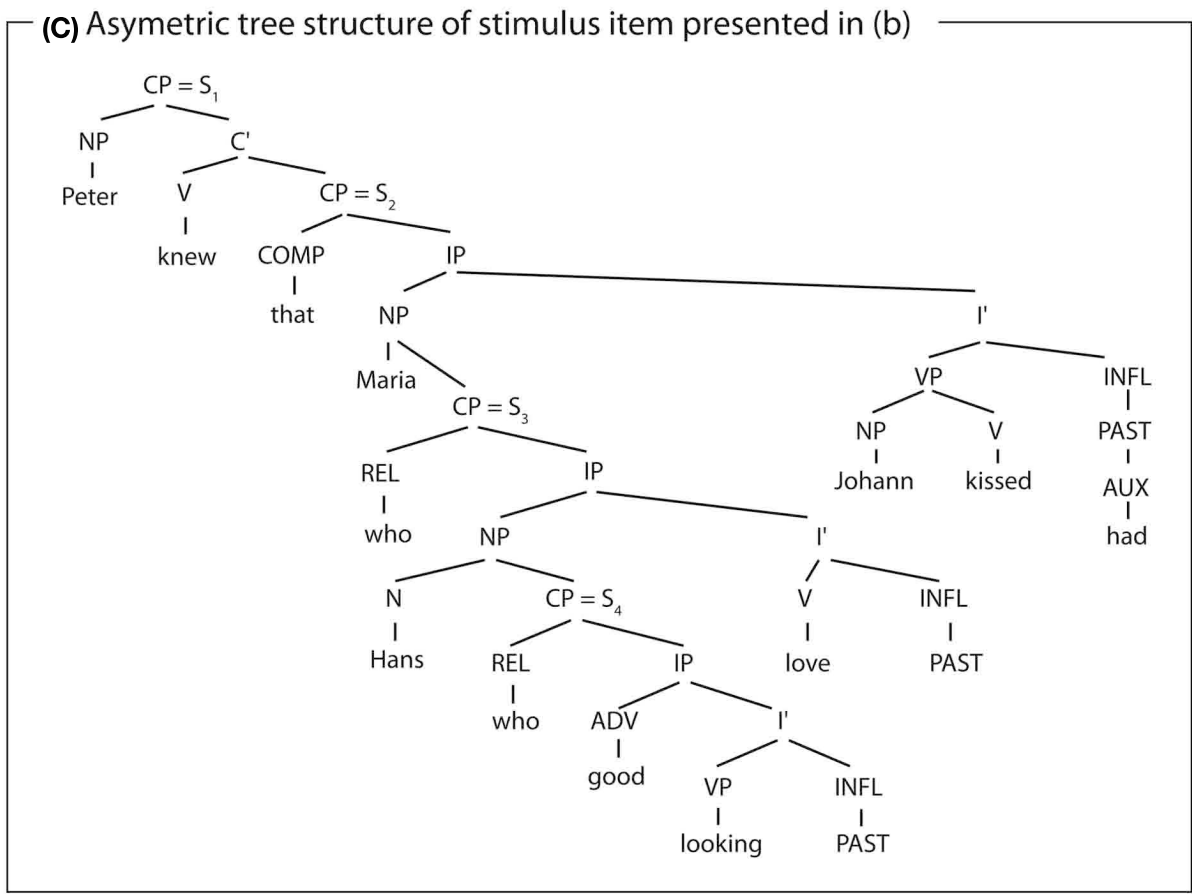

FIGURE 3 | Structure and examples of German sentences used in Makuuchi et al. (2009). (A) Schematic view of non-embedded and embedded structures described symmetrically. (B) Example sentence of embedded structure. (C) Tree structure (asymmetric) for example sentence displayed in
(B). Note that although the structure of the sentence displayed in Figure $\mathbf{3 C}$ could be described schematically as a symmetrical one (Figures $\mathbf{3 A}, \mathbf{B}$ ), this is not an adequate description since natural grammars contain functional categories requiring an asymmetrical description (Figure $\mathbf{3 C}$ ). 
For songbirds, the argument concerning the mechanism underlying grammar learning is different. In songbirds, the ability to learn grammatical sequences is based on a brain system mediating auditory input-to-vocal output (Fujimoto et al., 2011, for a review, see Bolhuis et al., 2010). This auditory-to-motor circuit, which probably acts in concert with a memory component, may underlie songbirds' ability to learn symmetric hierarchies (Bloomfield et al., 2011). In humans, the dorsal fiber bundle that connects the sensory auditory cortex to the premotor cortex can be viewed as a candidate neural structure of a functionally parallel auditory-to-motor circuit. This structure appears to play a crucial role in phonologybased language learning in humans during early infancy (Berwick et al., 2011).

\section{LANGUAGE DEVELOPMENT AND BRAIN MATURATION IN HUMANS}

In the past, the dorsal pathway that connects the temporal cortex to the frontal cortex, as observed in adults (Catani et al., 2002), has been proposed to not only support auditory-to-motor mapping (Hickok and Poeppel, 2007; Saur et al., 2008) but also to subserve the processing of syntactically complex sentences (Friederici et al., 2006; Friederici, 2009). Both views are evidenced by data from patient studies. On the one hand, lesions of the dorsal pathway result in conduction aphasia which is characterized by the inability to repeat speech (Geschwind, 1965a,b). On the other hand, lesions of the dorsal pathway correlate with deficits in syntactic processing (Wilson et al., 2011). Unfortunately, however, these patient studies do not allow a functional segregation of different parts of the dorsal pathway. Therefore, ontogenetic data may provide relevant information.

Newborns and infants show impressive language learning abilities. Newborns learn simple grammatical rules from auditory input after brief exposure (Gervain et al., 2008; Teinonen et al., 2009). By the age of 4 months, infants can learn the rule-based dependency of non-adjacent elements in a novel natural language, again after brief exposure to correct sentences (Friederici et al., 2011). During production, very young infants demonstrate a language-specific prosody in their cry patterns, long before they start to babble (Mampe et al., 2009), and during the babbling phase, they continuously tune their production toward the phonology of their target language (de Boysson-Bardies et al., 1984). This early phonology-based learning stage should require a circuit allowing auditory-to-motor mapping. Structural imaging data shows that newborns display such an auditory-to-motor circuit in the form of a dorsal pathway which links the temporal cortex to the premotor cortex (Perani et al., 2011). This dorsal pathway connecting to the premotor cortex must be separated from an additional dorsal pathway that connects the temporal cortex to Broca's area which is present in adults but not myelinized in infants (see Figure 4).

Here, it is proposed that there are two functionally distinct parts of the dorsal pathway (see Figure 4): one part connecting the temporal cortex to the premotor cortex (hereafter called Dorsal Pathway I) and a second, more medially located part, connecting the temporal cortex to Broca's area (hereafter called Dorsal Pathway II).

Dorsal Pathway I, supporting sensory-to-motor mapping, is present at birth, whereas Dorsal Pathway II is not (Perani et al., 2011). Previous studies with infants between 1 and 4 months old suggested that the dorsal pathway connecting to Broca's area is present early in life (Dubois et al., 2006, 2009), although the data appear to indicate that only the part of the dorsal pathway which connects to the premotor cortex (Dorsal Pathway I) is present. The authors proposed this may be due to methodological problems

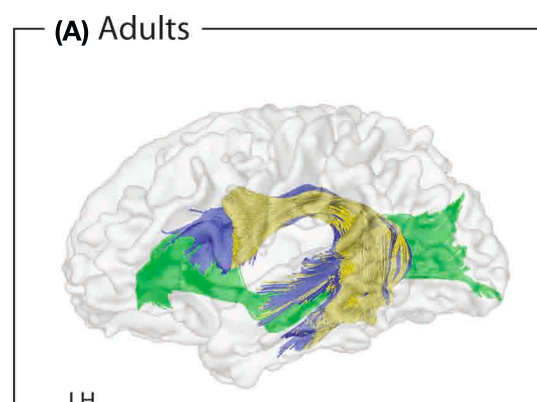

LH

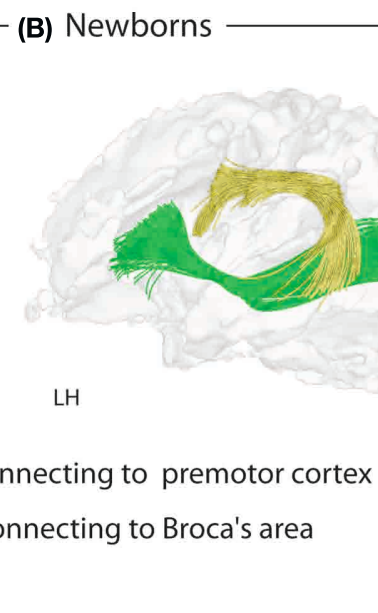

Adapted from Perani et al., PNAS, 2011

FIGURE 4 | Fiber tracking of diffusion tensor imaging data with seed in Broca's area and seed in the precentral gyrus/premotor cortex in (A) adults and (B) newborns. Two parts of the dorsal pathway are present in adults; one connecting the temporal cortex via the fasciculus arcuatus (AF) and the superior longitudinal fasciculus (SLF) to the inferior frontal gyrus, i.e., Broca's area (blue), and one connecting the temporal cortex via the AF/SLF to the precentral gyrus, i.e., premotor cortex (yellow). In newborns, only the part connecting to the precentral gyrus can be detected. The ventral pathway connecting the ventral inferior frontal gyrus via the extreme capsule fiber to the temporal cortex (green) is detectable in adults and newborns. LH, left hemisphere. 
and base their argumentation on functional data showing that Broca's area is activated in response to speech at this age (DehaeneLambertz et al., 2010). The study on newborns, however, indicates that this argumentation must be challenged, since for newborns although they show activation in Broca's area, neither a functional nor a structural connectivity between Broca's area and the temporal cortex can be found (Perani et al., 2011). Thus, it remains to be demonstrated whether the connection between the temporal cortex and Broca's area is fully present in infants between 1 and 4 months old, in particular, since another recent study reports that the dorsal pathway is limited to the parietal and temporal sections in early infancy (Leroy et al., 2011). At least, the data from Perani et al. (2011) indicate that in newborns, only the dorsal pathway that connects to the premotor cortex is myelinized. This connection may not only support tuning processes during babbling, but, moreover, the observed early learning of rule-based dependencies from auditory input in human infants (Gervain et al., 2008; Friederici et al., 2011; Kudo et al., 2011).

Dorsal Pathway II, connecting the temporal cortex to Broca's area, only develops as the brain matures, and is not even fully myelinized at the age of seven (Brauer et al., 2011; see Figure 5). It is argued that Dorsal Pathway II supports the processing of multi-level hierarchically structured sentences. The argument is based on two additional, independent findings. First, adults activate Broca's area and the posterior portion of the superior temporal gyrus and sulcus when processing syntactically complex sentences (Bornkessel et al., 2005; Friederici et al., 2009). Second, only adults with a mature Dorsal Pathway II are able to process syntactically complex sentences correctly, whereas children under the age of seven - an age at which the Dorsal Pathway II is not yet fully matured - are not (Hahne et al., 2004; Dittmar et al., 2008).

These findings in humans make it likely that grammatical rule learning and processing in infants and in adults are partly based on different brain structures. Learning and processing of auditory structured sequences, as shown in infants, could be based on the ability to identify phonological statistical relations of elements in a sequence and some memory capacity. This ability may partly be based on Dorsal Pathway I. In adults, this automatic way of learning from the auditory input is no longer at work, and strategic processes take over (Mueller et al., 2010).

Finally, the question remains: What is the function of the ventral pathway in language processing? The connection between the anterior portion of the prefrontal cortex and the middle temporal gyrus via the extreme capsule fiber system has been related functionally to semantic processing and comprehension (Saur et al., 2008; Tyler and Marslen-Wilson, 2008). There is accumulating evidence in support of the view that this pathway is relevant for semantic processes (for a review, see Weiller et al., 2011). However, the ventral pathway also appears to be involved in the processing of some aspects of syntax (Friederici et al., 2006; Tyler et al., 2011). The data on this issue are sparse, but it seems that the processing of simple sentences (e.g., The pilot is flying the plane) does not necessarily recruit the posterior portion of Broca's area, and thus the dorsal pathway (Saur et al., 2008; Tyler and Marslen-Wilson, 2008). The finding that the ventral pathway supports the computation of semantic relations, as well as some syntactic dependencies, raises the question of whether there is a general underlying mechanism capturing both aspects, or whether one has to assume two ventral pathways. Future studies will have to resolve this issue.

\section{CONCLUSION}

In light of these across species and within-human findings, we can speculate that there is a parallel mechanism for sequence learning across species, which is based on an auditory (input)-tomotor (output) circuit. In songbirds, the causal relation between the auditory input-to-vocal output and sequence learning is well established (Scharff and Nottebohm, 1991; Fujimoto et al., 2011). In humans, a respective neural circuit - Dorsal Pathway I connecting the temporal cortex to the premotor cortex - is present at birth and may be responsible for rule-based sequence learning observed in young infants (Teinonen et al., 2009; Kudo et al., 2011). In adults,

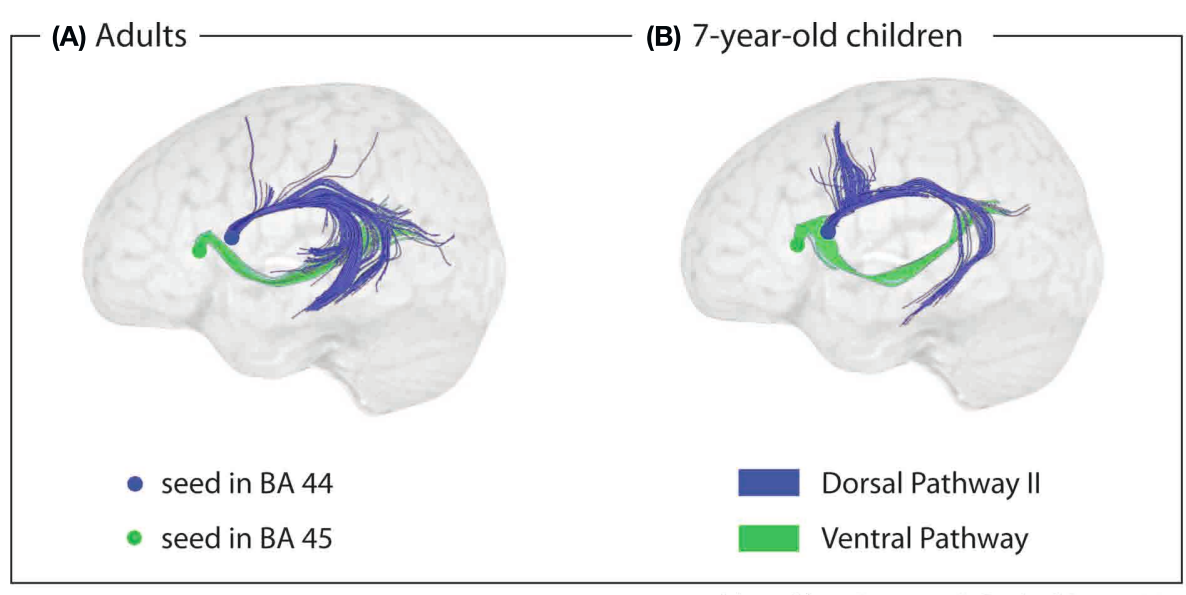

Adapted from Brauer et al., Cerebral Cortex, 2011

FIGURE 5 | Fiber tracking of diffusion tensor imaging data with seeds in Brodmann Area (BA) $\mathbf{4 4}$ and $\mathbf{4 5}$ in (A) adults and (B) 7-year-old children. The dorsal pathway connects the posterior part of Broca's area (BA 44) to the temporal cortex via the AF/SLF. The ventral pathway connects the anterior part of Broca's area (BA 45) to the temporal cortex via the extreme capsule fiber system. 
this neural network appears to support bottom-up processes such as speech perception and repetition (Saur et al., 2008).

However, Dorsal Pathway II, connecting Broca's area to the temporal cortex, may specifically subserve the processing of languagelike hierarchical structures. The supporting evidence for this is twofold: first, the dorsal pathway is generally stronger in human adults than in non-human primates (Rilling et al., 2008), and second, Dorsal Pathway II, in particular, is not myelinized at birth

\section{REFERENCES}

Abe, K., and Watanabe, D. (2011). Songbirds possess the spontaneous ability to discriminate syntactic rules. Nat. Neurosci. 14, 1067-1074.

Anwander, A., Tittgemeyer, M., von Cramon, D. Y., Friederici, A. D., and Knösche, T. R. (2007). Connectivity-based parcellation of Broca's area. Cereb. Cortex 17, 816-825.

Bahlmann, J., Schubotz, R., and Friederici, A. D. (2008). Hierarchical artificial grammar processing engages Broca's area. NeuroImage 42, 525-534.

Berwick, R. C., Okanoya, K., Beckers, G. J. L., and Bolhuis, J. J. (2011). Songs to syntax: the linguistics of birdsong. Trends Cogn. Sci. (Regul. Ed.) 15, 113-121.

Bloomfield, T. C., Gentner, T. Q., and Margoliash, T. (2011). What birds have to say about language. Nat. Neurosci. 14, 947-948.

Bolhuis, J. J., Okanoya, K., and Scharff, C. (2010). Twitter evolution: converging mechanisms in birdsong and human speech. Nat. Rev. Neurosci. $11,747-759$.

Bornkessel, I., Zyssett, S., Friederici, A. D., von Cramon, D. Y., and Schlesewsky, M. (2005). Who did what to whom? The neural basis of argument hierarchies during language comprehension. Neuroimage 26, 221-233.

Brauer, J., Anwander, A., and Friederici, A. D. (2011). Neuroanatomical prerequisites for language functions in the maturing brain. Cereb. Cortex 21, 459-466.

Catani, M., Howard, R. J., Pajevic, S., and Jones, D. K. (2002). Virtual in vivo interactive dissection of white matter fasciculi in the human brain. Neuroimage 17, 77-94.

Chomsky, N. (1995). The Minimalist Program. Cambridge, MA: MIT Press.

de Boysson-Bardies, B., Sagart, L., and Durant, C. (1984). Discernible differences in the babbling of infants according to target language. J. Child Lang. 11, 1-15. de Vries, M. H., Monaghan, P., Knecht, S., and Zwitserlood, P. (2008). Syntactic structure and artificial grammar learning: the learnability of embedded hierarchical structures. Cognition 107, 763-774.

Dehaene-Lambertz, G., Hertz-Pannier, L., Dubois, J., Mériaux, S., Roche, A., Sigman, M., and Dehaene, S. (2010). Functional organization of perisylvian activation during presentation of sentences in preverbal infants. Proc. Natl. Acad. Sci. U.S.A. 103, 14240-14245. E., and Tomasello, M. (2008). German children's comprehension of word order and case marking in causative sentences. Child Dev. 79, 1152-1167.

Dubois, J., Hertz-Pannier, L., Cachia, A., Mangin, J. F., Le Bihan, D., and Dehaene-Lambertz, G. (2009). Structural asymmetries in the infant language and sensorimotor networks. Cereb. Cortex 19, 414-423.

Dubois, J., Hertz-Pannier, L., DehaeneLambertz, G., Cointepas, Y., and Le Bihana, D. (2006). Assessand maturation of infants' cerebral white matter fiber bundles: a feasibility study using quantitative diffusion tensor imaging and tractography. Neuroimage 30, 1121-1132.

Fitch, W. T., and Hauser, M. D. (2004). Computational constraints on syntactic processing in a nonhuman primate. Science 303, 377-380.

Friederici, A. D. (2009). Pathways to language: fiber tracts in the human brain. Trends Cogn. Sci. (Regul. Ed.) 13, 175-181.

Friederici, A. D., Bahlmann, J., Heim, S., Schubotz, R. I., and Anwander, A. (2006). The brain differentiates human, and non-human grammars: functional localization, and structural connectivity. Proc. Natl. Acad. Sci. U.S.A. 103, 2458-2463.

Friederici, A. D., Makuuchi, M., and Bahlmann, J. (2009). The role of
Dittmar, M., Abbot-Smith, K., Lieven, ment of the early organization

and only fully develops at around the time children master syntactically complex sentences (Brauer et al., 2011; Perani et al., 2011). In adults, this pathway may be involved in fronto-to-temporal top-down predictive processes during language comprehension.

\section{ACKNOWLEDGMENTS}

I thank the two reviewers and Jens Brauer for helpful comments on the manuscript.

the posterior superior temporal cortex in sentence comprehension. $\mathrm{Neu}$ roreport 20, 563-568.

Friederici, A. D., Müller, J., and Oberecker, R. (2011). Precursors to natural grammar learning: preliminary evidence from 4-month-old infants. PLoS ONE 6, e17920. doi:10.1371/journal.pone.0017920

Fujimoto, H., Hasegawa, T., and Watanabe, D. (2011). Neural coding of syntactic structure in learned vocalizations in the songbird. J. Neurosci. 31 , 10023-10033.

Gentner, T. Q., Fenn, K. M., Margoliash, D., and Nusbaum, H. C. (2006). Recursive syntactic pattern learning by songbirds. Nature 440 1204-1207.

Gervain, J., Macagno, F., Cogoi, S., Pen, A. M., and Mehler, J. (2008). The neonate brain detects speech structure. Proc. Natl. Acad. Sci. U.S.A. 105 14222-14227.

Geschwind, N. (1965a). Disconnexion syndromes in animals and man. I. Brain 88, 237-294.

Geschwind, N. (1965b). Disconnexion syndromes in animals and man. II. Brain 88, 585-644.

Hahne, A., Eckstein, K., and Friederici, A. D. (2004). Brain signatures of syntactic and semantic processes during children's language development. J. Cogn. Neurosci. 16, 1302-1318.

Hauser, M., Chomsky, N., and Fitch, W. (2002). The faculty of language: what is it, who has it, and how did it evolve? Science 298, 1569-1579.

Hickok, G., and Poeppel, D. (2007). The cortical organization of speech perception. Nat. Rev. Neurosci. 8, 393-402.

Kudo, N., Nonaka, Y., Mizuno, N., Mizuno, K., and Okanoya, K. (2011), On-line statistical segmentation of a non-speech auditory stream in neonates as demonstrated by eventrelated brain potentials. Dev. Sci. 14 1100-1106.

Leroy, F., Glasel, H., Dubois, J., HertzPannier, L., Thirion, B., Mangin, J.-F., and Dehaene-Lambertz, G. (2011). Early maturation of the linguistic dorsal pathway in human infants. J. Neurosci. 31, 1500-1506.

Makris, N., and Pandya, D. (2009). The extreme capsule in humans and rethinking of the language circuitry. Brain Struct. Funct. 213, 343-358.

Makuuchi, M., Bahlmann, J., Anwander, A., and Friederici, A. D. (2009). Segregating the core computational faculty of human language from working memory. Proc. Natl. Acad. Sci. U.S.A. 106, 8362-8367.

Mampe, B., Friederici, A. D. Christophe, A., and Wermke, K. (2009). Newborns' cry melody is shaped by their native language. Curr. Biol. 19, 1994-1997.

Mueller, J. L., Bahlmann, J., and Friederici, A. D. (2010). Learnability of embedded syntactic structures depends on prosodic cues. Cogn. Sci. 34, 338-349.

Perani, D., Saccuman, M. C., Scifo, P., Anwander, A., Spada, D., Baldoli, C., Poloniato, A., Lohmann, G., and Friederici, A. D. (2011). Neural language networks at birth. Proc. Natl. Acad. Sci. U.S.A. 108, 16056-16061.

Perruchet, P., and Rey, A. (2005). Does the mastery of center-embedded linguistic structures distinguish humans from nonhuman primates? Psychon. Bull. Rev. 12, 307-313.

Petrides, M., and Pandya, D. (2009). Distinct parietal and temporal pathways to the homologues of Broca's area in the monkey. PLoS Biol. 7, e1000170. doi:10.1371/journal.pbio. 1000170

Rilling, J. K., Glasser, M. F., Preuss, T. M., Ma, X. Y., Zhao, T. J., Hu, X. P., and Behrens, T. E. J. (2008). The evolution of the arcuate fasciculus revealed with comparative DTI. Nat. Neurosci. 11, 426-428.

Saur, D., Kreher, B. W., Schnell, S., Kümmerer, D., Kellmeyer, P., Vry, M. S. Umarova, R. M., Glauche, V., Abel, S., Huber, W., Rijntjes, M., Hennig, J., and Weiller, C. (2008). Ventral and dorsal pathways for language. Proc. Natl. Acad. Sci. U.S.A. 105, 18035-18040. 
Scharff, C., and Nottebohm, F. (1991). A comparative-study of the behavioral deficits following lesions of various parts of the zebra finch song systemimplications for vocal learning. J. Neurosci. 11, 2896-2913.

Teinonen, T., Fellman, V., Näätänen, R., Alku, P., and Huotilainen, M. (2009). Statistical language learning in neonates revealed by event-related brain potentials. BMC Neurosci. 10, 21. doi:10.1186/1471-2202-10-21

Tyler, L. K., and Marslen-Wilson, W. D. (2008). Fronto-temporal brain systems supporting spoken language comprehension. Philos. Trans. R. Soc. Lond. B Biol. Sci. 363, 1037-1054.

Tyler, L. K., Marslen-Wilson, W. D., Randall, B., Wright, P., Devereux, B. J., Zhuang, J., Papoutsi, and M., and Stamatakis, E. A. (2011). Left inferior frontal cortex and syntax: function, structure and behaviour in lefthemisphere damaged patients. Brain 134, 415-431.

Weiller, C., Bormann, T., Saur, D., Musso, M., and Rijntjes, M. (2011). How the ventral pathway got lost: and what its recovery might mean. Brain Lang. 118, 29-39.
Wilson, S. M., Galantucci, S., Tartaglia, M. C., Rising, K., Patterson, D. K., Henry, M. L., Ogar, J. M., DeLeon, J., Miller, B. L., and Gorno-Tempini, M. L. (2011). Syntactic processing depends on dorsal language tracts. Neuron 72, 397-403.

Conflict of Interest Statement: The author declares that the research was conducted in the absence of any commercial or financial relationships that could be construed as a potential conflict of interest.
Received: 06 October 2011; accepted: 18 January 2012; published online: 06 February 2012.

Citation: Friederici AD (2012) Language development and the ontogeny of the dorsal pathway. Front. Evol. Neurosci. 4:3. doi: 10.3389/fnevo.2012.00003

Copyright () 2012 Friederici. This is an open-access article distributed under the terms of the Creative Commons Attribution Non Commercial License, which permits non-commercial use, distribution, and reproduction in other forums, provided the original authors and source are credited. 